\title{
A Paradigm for Facial Skin Rejuvenation
}

\author{
Sean D. Doherty, M.D., ${ }^{1}$ Christy B. Doherty, M.D., ${ }^{1}$ Jodi S. Markus, M.D., ${ }^{1}$ \\ and Ramsey F. Markus, M.D. ${ }^{1}$
}

\section{ABSTRACT}

There is a significant desire by patients to reverse the signs of aging caused by photodamage. Numerous procedures for facial skin rejuvenation have been developed in an attempt to minimize the erythema, dyspigmentation, and rhytides associated with photoaging. The initial procedures developed for facial rejuvenation involve skin resurfacing via complete ablation of layers of skin. Of these procedures, ablative laser resurfacing is the most precise technique and is considered the gold standard for facial skin rejuvenation. Although ablative procedures are quite efficacious, they carry significant patient downtime and risks of adverse effects such as scarring and dyspigmentation. Concerns regarding patient morbidity have led to the development of nonablative procedures that target dermal collagen without damaging the epidermis. Of these technologies, intense pulsed light is the most commonly used because it effectively targets both the erythema and dyspigmentation seen in photoaging. Nonablative techniques minimize side effects and patient downtime; however, they do not match the results seen in fully ablative procedures. Fractional laser technologies-first nonablative and more recently ablative-represent the most recent attempt to match the results seen in fully ablative procedures with less patient downtime. Their results are promising but require further study.

KEYWORDS: Photodamaged, rejuvenation, resurfacing, laser, fractional

The considerable desire of patients to look youthful is matched only by the number of modalities offered to patients both medically and nonmedically to achieve that goal. Many of these technologies have been well studied, whereas others have not. It is important to an individual's psychological well-being to be content with his or her appearance; however, the cosmetic treatment of any patient should carefully be weighed against possible side effects. Likewise, procedures are most appropriately performed by individuals who are well trained to minimize risk to the patient.

Aging of the skin is likely caused by both intrinsic or biologic factors and extrinsic or environmental factors. These factors are interconnected and may share a final common pathway. ${ }^{1}$ The most important extrinsic factor in skin aging is ultraviolet (UV) radiation from the sun, but other environmental factors such as smoking, sleeping positions, and repetitive facial expressions can have an effect on skin appearance as well.

There are multiple processes involved in facial aging. The weakening and repositioning of soft tissue as well as soft tissue volume loss lead to excess skin and deep creases on the lower face and the exposure of bony prominences on the upper face. Finer lines and rhytides occur from repetitive facial movements as well as other intrinsic and extrinsic factors. Finally, the quality and appearance of the skin change over time, mainly secondary to photodamage. This change takes many forms but primarily involves erythema, abnormal pigmentation, and textural irregularities. Because aging of the
${ }^{1}$ Department of Dermatology, Baylor College of Medicine, Houston, Texas.

Address for correspondence and reprint requests: Ramsey F. Markus, M.D., Department of Dermatology, Baylor College of Medicine, 1709 Dryden, Suite 10.50, Houston, TX 77030 (e-mail: rmarkus@bcm.edu).
Management of the Aging Face; Guest Editor, Anthony P. Sclafani, M.D., F.A.C.S.

Facial Plast Surg 2009;25:245-251. Copyright (C) 2009 by Thieme Medical Publishers, Inc., 333 Seventh Avenue, New York, NY 10001, USA. Tel: +1(212) 584-4662.

DOI 10.1055/s-0029-1242036. ISSN 0736-6825. 
face can take many forms, it is important when first evaluating the patient to determine the aspects of their appearance that concerns them the most. An appropriate modality to target those concerns may then be chosen.

Although soft tissue augmentation, surgical intervention, and chemodeinnervation are important tools available in facial rejuvenation, they are beyond the scope of this article and will not be discussed here. Likewise, certain lasers used to target specific facial abnormalities such as telangiectasias or melasma will also not be discussed. Instead, we will examine those therapies that directly target the quality of aging facial skin. These include topical therapies as well as ablative and nonablative technologies.

\section{TOPICAL THERAPIES}

Photoaging results from chronic UV-induced skin damage superimposed on intrinsic skin aging and accounts for most age-associated changes in skin appearance. ${ }^{2} \mathrm{It}$ is more prominent in lighter skin types and on exposed areas, such as the face. ${ }^{1,3}$ Protecting against this UV damage by either decreased exposure or sunscreen use has been shown not only to improve skin appearance by decreasing photoaging but also to reverse some signs of aging already present. ${ }^{3,4}$ It is important that any sunscreen used protects against both UVB and the longer-wavelength UVA rays. This can be achieved through either newer organic (chemical) agents or inorganic (physical) blockers. ${ }^{2}$

Retinoids are considered the mainstay for topical treatment and prevention of photoaging. They are vitamin A derivatives and can be either natural or synthetic. Certain retinoids are registered as drugs, including tretinoin, alitretinoin, tazarotene, and adapalene. Other retinoids, such as retinol, are considered cosmeceuticals (medically active cosmetics). Tretinoin is the most studied topical retinoid and has been proved to clinically and biochemically repair photodamaged skin. The side effects of treatment are generally mild and most commonly involve redness and irritation that can be controlled by emollients and decreased frequency of application. It is very important to combine use with appropriate sunscreens as retinoids increase the sun-sensitivity of skin, and UV radiation downregulates the retinoid receptor in the skin, leading to decreased efficacy. ${ }^{5}$

The skin contains both enzymatic and nonenzymatic antioxidants. These provide protection from reactive oxygen species formed during normal cellular processes. Excessive UV radiation is thought to deplete this antioxidant supply, and one theory of photoaging is that the resultant increased oxidative stress helps to mediate what is seen clinically as aging. ${ }^{1}$ On the basis of this theory, numerous antioxidants have been studied for their potential to reduce the signs of aging, and many are marketed to consumers in the form of cosmeceuticals. These topical therapies range from topical vitamin $\mathrm{C}$ preparations to extracts from teas and plants. The range of these new therapies is rapidly expanding due to both the availability of new products and the financial rewards for developing a successful product. ${ }^{6}$ Most of these cosmeceuticals show minimal benefit, and their risk of side effects is similarly quite low. Further studies are needed to fully assess their place in facial skin rejuvenation.

\section{FULLY ABLATIVE THERAPIES}

The first techniques developed for facial skin rejuvenation were ablative resurfacing procedures. These procedures completely destroy layers of skin and include chemical peels, mechanical abrasion, and lasers. In general, a thorough understanding of skin anatomy, normal wound healing, and the histology of the underlying pathology allows an appropriate treatment to be selected. Photodamaged skin has been classified by Rubin ${ }^{7}$ based on the histologic depth of the clinical changes. A treatment modality should be chosen that goes at least as deep as the deepest skin problem to achieve optimal results. ${ }^{8}$ The rejuvenative effects are proportionate to the depth of penetration but so are the potential risks and patient morbidity. Ablative treatments should be performed only by individuals skilled in the technique. If the performing physician is well trained, the chosen modality should not make too much of a difference in terms of patient outcome. The one exception to this statement may be ablative laser therapy. The ablative laser has the ability to very precisely control the depth of tissue ablated with each pass and may in fact be superior to the other ablative modalities.

Chemical peeling is the process of applying chemicals to the skin to destroy the outer damaged layers; peels can be divided into three categories based on their depth of penetration. Superficial peels destroy part or all of the epidermis and are mainly indicated for improvement in skin texture and superficial dyschromias. ${ }^{9}$ They include $\alpha$-hydroxy acid (glycolic acid) peels ${ }^{10}$ salicylic acid peels,${ }^{11}$ tretinoin peels, ${ }^{12}$ resorcinol peels, $10 \%$ to $20 \%$ trichloroacetic acid (TCA) peels, ${ }^{8}$ and Jessner solution (salicylic acid, resorcinol, lactic acid, and ethanol) peels. ${ }^{8}$ The superficial peels have minimal downtime, modest results, and may require serial treatments or a combination with other modalities.

Medium-depth peels destroy the entire epidermis and part or all of the papillary dermis; they primarily target dyschromia (mainly solar lentigines) and textural changes of the skin. ${ }^{9}$ These peels include $35 \%$ TCA peels and higher-concentration glycolic acid peels. There are also numerous combination peels that are medium depth. Some examples are Monheit's 
combination (Jessner's solution with $35 \%$ TCA), ${ }^{13}$ Brody's combination (icing skin with solid carbon dioxide prior to TCA peel) ${ }^{14}$ and Coleman's combination (glycolic acid 70\% is applied before application of TCA). ${ }^{15}$

Deep peels destroy the epidermis and papillary dermis and extend into the reticular dermis; they are generally used for dyschromia, fine and coarse wrinkles, and acne scars. Their depth of penetration creates maximal effect for the production of new collagen. ${ }^{9}$ However, as mentioned above, the deep depth of penetration may lead to significant patient morbidity and risk of complication. Also, the liquid nature of the peels can make uniform ablation difficult, requires significant defatting of the skin, and can drip onto areas that are not intended to be treated. Deep peels are composed of phenol and croton oil in various combinations. ${ }^{9}$ The deep phenol-based peels must be performed with full cardiopulmonary monitoring and intravenous sedation or regional blocks. ${ }^{9}$

Microdermabrasion and dermabrasion are both techniques that mechanically remove layers of skin. Microdermabrasion is more superficial than dermabrasion but has been shown to improve facial photodamage. ${ }^{16}$ The minimal improvements are primarily in skin texture and are very transient. Multiple methods of microdermabrasion exist, but the most commonly employed method uses a machine that blows aluminum oxide crystals over the skin to remove thin layers. ${ }^{17}$ The technique can be performed very quickly and has minimal or no patient downtime. Mechanical dermabrasion generally penetrates much more deeply and can be used to target rhytides and scars. This technique usually involves a mechanical diamond-studded fraise, wire brushes, or sandpaper. With mechanical dermabrasion, the operator has direct control over the depth of injury. However, the practice of dermabrasion is very much an art; outcome, uniformity of results, and side effects, including potential deep gouges and scars, are very dependent upon operator experience and technique. ${ }^{18}$

The most precise technique for skin resurfacing is fully ablative laser treatment. These lasers act by targeting intracellular water and include the carbon dioxide $\left(\mathrm{CO}_{2} ; 10,600 \mathrm{~nm}\right)$ and the erbium:yttrium-aluminumgarnet (Er:YAG; 2930 to $2940 \mathrm{~nm}$ ) lasers. ${ }^{3}$ The $\mathrm{CO}_{2}$ laser is considered the "gold standard" for facial skin resurfacing. ${ }^{1}$ Each pass of the laser precisely ablates layers of photodamaged skin, plateauing at a maximum penetration depth of $\sim 250 \mu \mathrm{m}$. This maximum depth ablates both the epidermis and superficial portion of the papillary dermis. ${ }^{19}$ Resurfacing with the $\mathrm{CO}_{2}$ laser typically produces at least a $50 \%$ improvement in overall skin tone and wrinkle severity. ${ }^{1}$ However, the fully ablative $\mathrm{CO}_{2}$ laser carries significant patient downtime until reepithelialization occurs. This can take at least 1 week, and complete recovery may require 1 month or more. ${ }^{1}$ Full ablation of the epidermis and superficial dermis can lead to complications such as infection, edema, erythema, and dyspigmentation. ${ }^{3}$

The Er:YAG laser is a fully ablative laser that has a higher affinity for water and, therefore, a smaller depth of penetration. The Er:YAG laser was developed to reduce the morbidity associated with $\mathrm{CO}_{2}$ laser resurfacing and has been shown to have similar results with equally aggressive treatment (Fig. 1). ${ }^{1} \mathrm{~A}$ single pulse of the Er:YAG laser at $5 \mathrm{~J} / \mathrm{cm}^{2}$ will ablate 20 to $25 \mu \mathrm{m}$ of tissue. It has a cleaner ablation than that of the $\mathrm{CO}_{2}$ laser (i.e., less coagulation of tissue) resulting in more bleeding after the procedure, a major reason for less depth of treatment resulting in less wrinkle improvement than with $\mathrm{CO}_{2}$. More powerful versions of the laser allow for up to $100 \mu \mathrm{m}$ of vaporization in addition to $25 \mu \mathrm{m}$ of coagulation to achieve a combination of traditional $\mathrm{CO}_{2}$ and Er:YAG effect.

\section{NONABLATIVE THERAPIES}

All ablative therapies carry a certain morbidity for patients, and many require significant patient downtime. Attempts to minimize risk and recovery time have led to the development of less-ablative or nonablative techniques for facial skin rejuvenation. These techniques use light or thermal energy to damage dermal collagen, leading to subsequent reactive collagen synthesis, while preserving the integrity of the epidermis. They can provide many cosmetic benefits, including improvement of skin texture, discoloration, and preexistent scarring, with minimal patient downtime. ${ }^{3}$

One of the most commonly used modalities for facial skin rejuvenation is intense pulsed light (IPL). IPL systems are high-intensity light sources that, unlike lasers, emit noncoherent light with a broad wavelength spectrum (515 to $1200 \mathrm{~nm}){ }^{20}$ The IPL has shown utility in the treatment of both the hyperpigmentation and erythema associated with photoaging, leading to a more even skin tone overall (Fig. 2). IPL achieves this improvement by targeting both melanin and hemoglobin. Significant changes in skin texture and rhytides have proved less achievable with the IPL alone. ${ }^{20}$ Erythema, blisters, and crusting are the most commonly reported side effects, and dyspigmentation and scarring have been reported, mostly in patients with darker skin types. ${ }^{20}$

Nonablative lasers with wavelengths in the visible light range of the electromagnetic spectrum target specific changes associated with aging. The pulsed dye laser $(585 \mathrm{~nm})$, the long-pulsed dye laser $(595 \mathrm{~nm})$, and the pulsed potassium phosphate laser $(532 \mathrm{~nm})$ target vascular lesions, whereas the $\mathrm{Q}$-switched alexandrite $(755 \mathrm{~nm})$ and diode $(800 \mathrm{~nm})$ lasers target pigmented lesions such as lentigines and nevi. ${ }^{21}$ Because of the enormous scope of this subject, these lasers will not be addressed in detail here. 

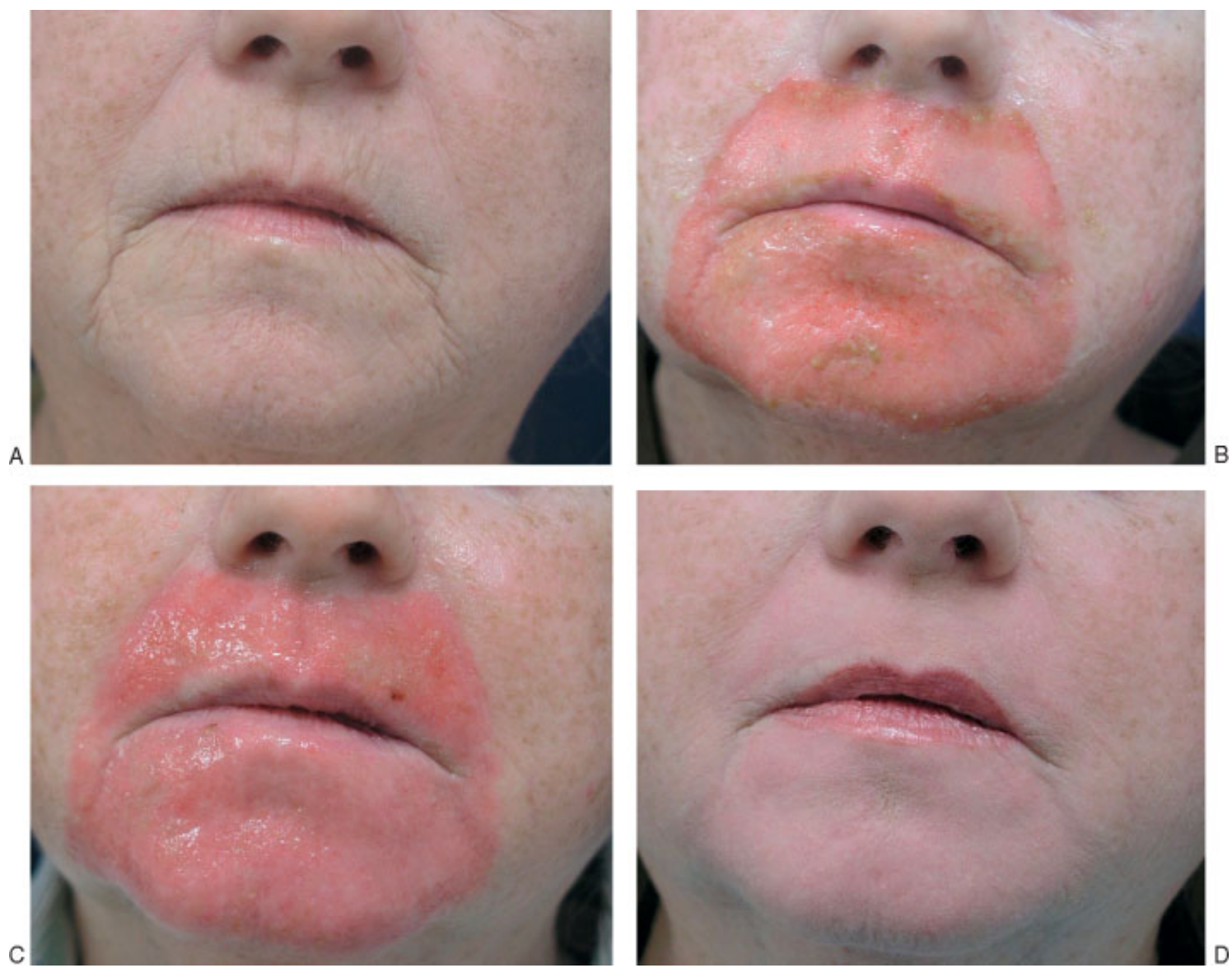

Figure 1 (A) Before erbium. (B) Day 3 after erbium. (C) Day 7 after erbium. (D) 3 months after erbium.

Nonablative lasers with longer wavelengths in the infrared (IR) range of the electromagnetic spectrum, such as the neodymium doped: yttrium aluminum garnet (Nd:YAG; $1064 \mathrm{~nm})$ and diode $(1450 \mathrm{~nm})$ lasers, selectively induce thermal injury to the papillary and upper reticular dermis without damaging the epidermis. ${ }^{3}$ These lasers employ cooling mechanisms to protect the epidermis from thermal injury. The infrared lasers have demonstrated very modest results in the treatment of mild to moderate rhytides, but they also carry only minimal patient risk and downtime.

Another nonablative therapy for facial skin rejuvenation is photodynamic therapy (PDT). PDT requires a photosensitizer, light, and oxygen. The most commonly
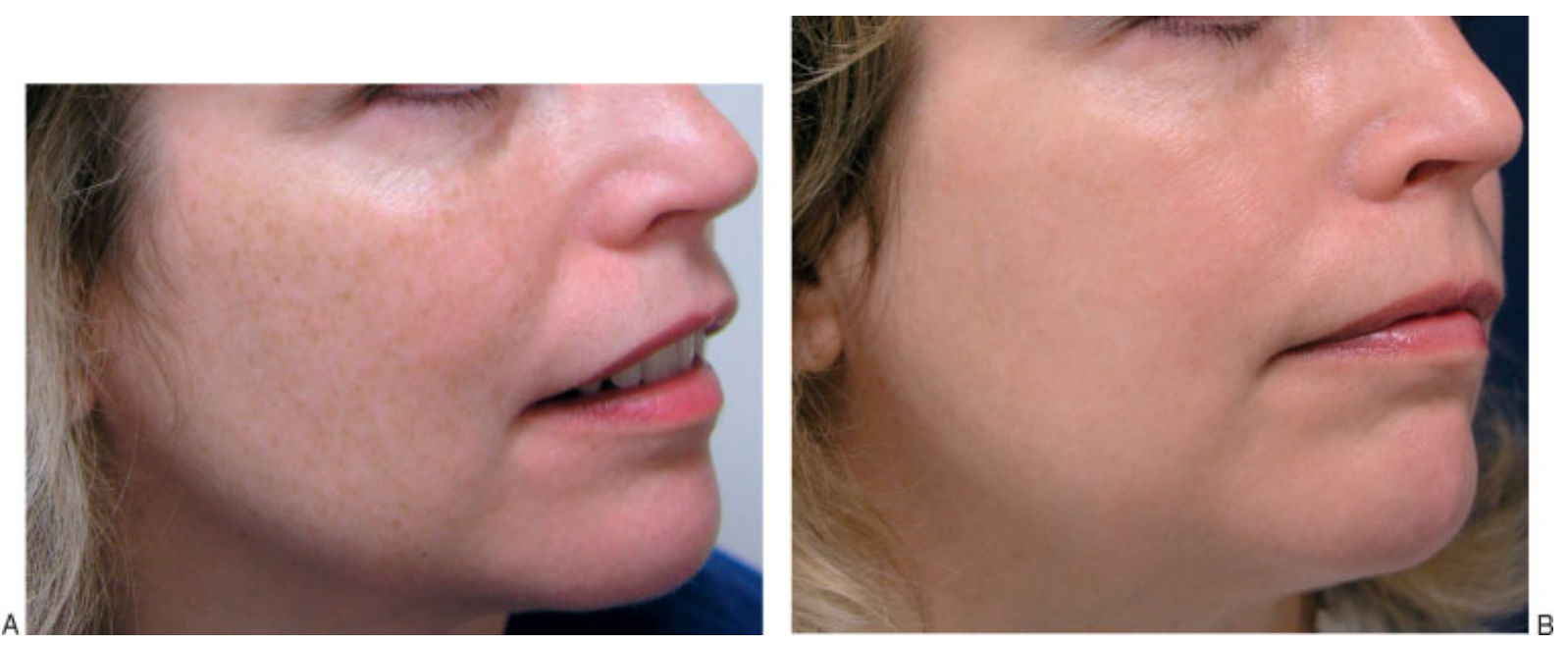

Figure 2 (A) Before IPL treatment. (B) After two IPL treatments. 

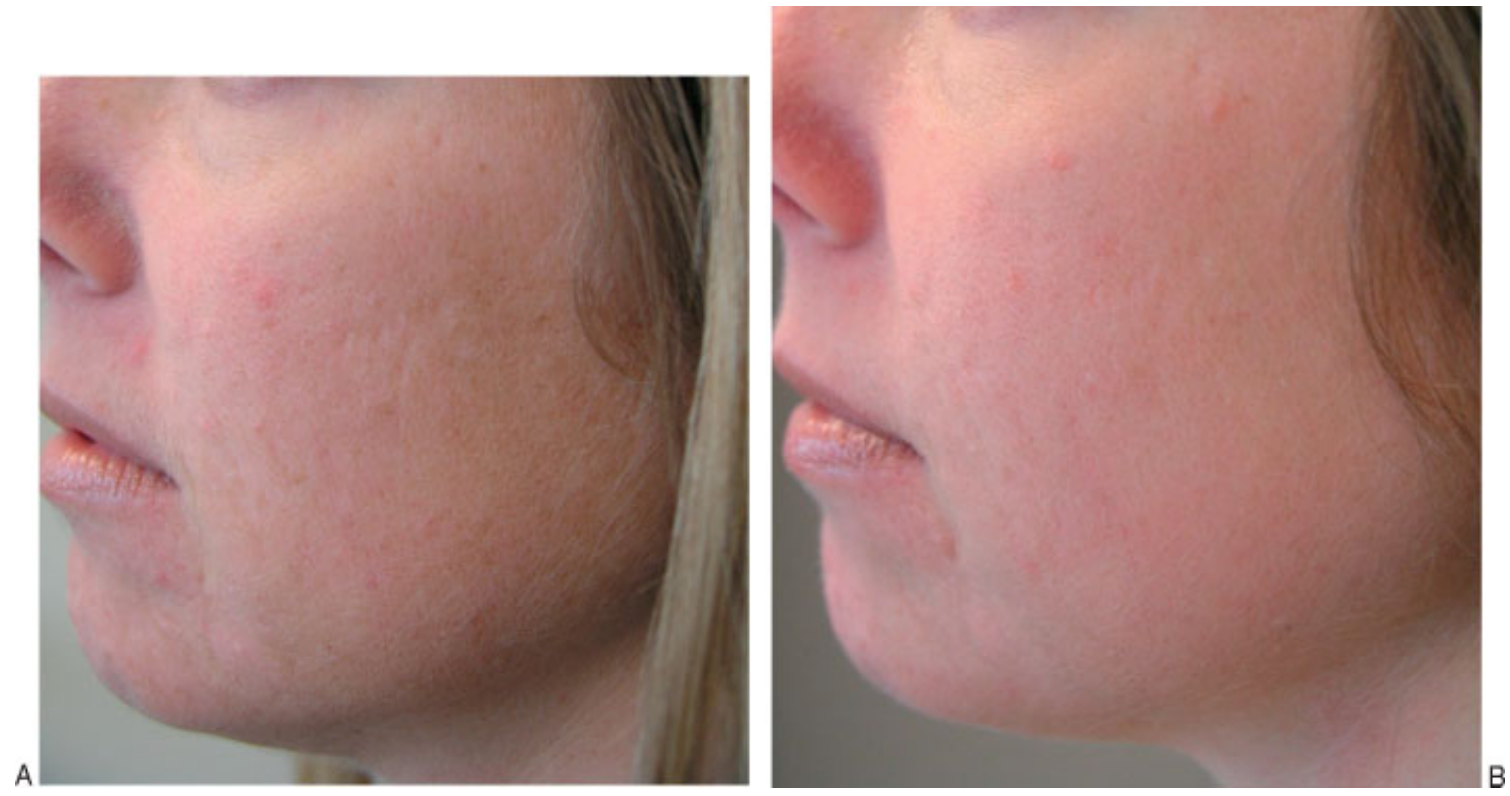

Figure 3 (A) Before nonablative treatment. (B) After three nonablative fractional resurfacing treatments.

used photosensitizer is 20\% 5-aminolevulinic acid (ALA). After application of the photosensitizer and an appropriate incubation time, ALA is converted to protoporphyrin IX and can be targeted by light. Blue light has classically been used in PDT because it targets the largest absorption band of protoporphyrin IX, known as the Soret band ${ }^{22}$; however, when used for photorejuvenation, a variety of other light sources, including red light and lasers, have been used. Multiple studies have demonstrated significant photorejuvenation with PDT, including appreciable decreases in erythema, dyspigmentation, and fine wrinkles, ${ }^{22}$ though how much is due to the laser or IPL device versus the PDT effect is debated.

Lastly, technologies employing heat or radiofrequency technologies have been used in nonablative facial skin rejuvenation. Radiofrequency devices generate heat through inherent resistance in dermal tissues, ${ }^{23}$ and nonlaser infrared light devices use controlled infrared light (1100 to $1800 \mathrm{~nm}$ ) to selectively heat the dermis. ${ }^{3}$ These technologies have been shown to result in mod- erate skin tightening, likely secondary to collagen contraction after synthesis and remodeling. The results have been disappointing, but the reported side effects are minimal and include erythema and burning. ${ }^{1}$

\section{FRACTIONAL PHOTOTHERMOLYSIS}

Although the nonablative therapies carry significantly less patient morbidity and downtime than that of fully ablative therapies, they do not routinely deliver the same results as ablative modalities (Figs. 3 and 4). The desire for a more consistently efficacious treatment without significant potential for adverse events and patient downtime led to the development of fractional photothermolysis. This concept is a variation of classic laser therapy whereby microscopic treatment zones (MTZs) of controlled width, depth, and density are created in the skin. These controlled zones of tissue damage are surrounded by viable tissue that allows for rapid repair of the treatment zones. ${ }^{24}$
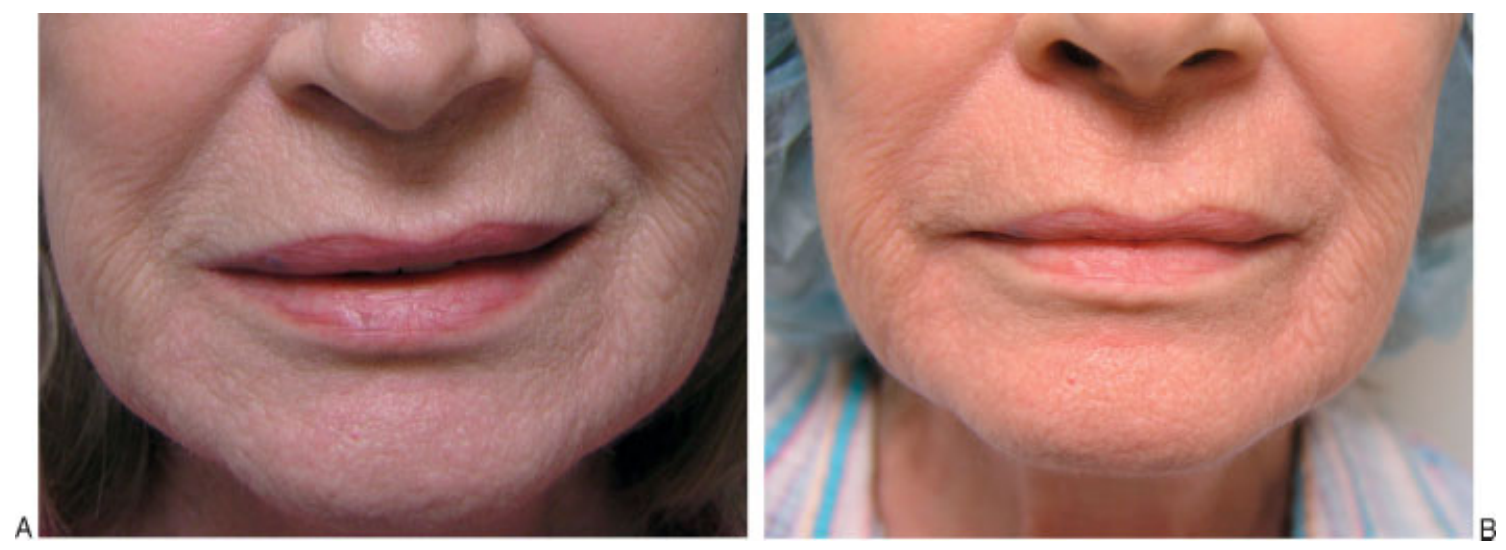

Figure 4 (A) Before treatment. (B) After three nonablative fractional resurfacing sessions with no improvement in wrinkles. 
Initially, only devices that relied on nonablative fractional photothermolysis were available. The first fractional device approved for clinical use consists of an erbium-doped fiber laser operating at a wavelength of $1550 \mathrm{~nm}$ that targets water as its chromophore. The laser may be operated in scanning mode via use of disposable roller tips, allowing for uniformly deposited MTZs despite variable hand speeds. Multiple passes can be made over the skin, and epidermal cooling is not required. For patient comfort, however, cooling devices such as forced air are often used in conjunction with topical anesthesia. Nonablative fractional photothermolysis has been shown to successfully treat photodamage and rhytides with only minimal side effects and limited patient downtime. ${ }^{25}$

Fractional delivery systems for ablative lasers, including $\mathrm{CO}_{2}$ and Er:YAG, have also been developed in an attempt to match the clinical results achieved with traditional ablative lasers. These devices cause microscopic zones of complete epidermal ablation with variable amounts of dermal ablation. As in nonablative fractional treatment, the MTZs are surrounded by normal skin leading to more rapid recovery with fewer side effects than that of conventional ablative lasers. ${ }^{24}$ The ablative fractional treatment commonly leads to serosanguineous discharge, and 1 to 2 days of wound care are required; however, rapid reepithelialization usually occurs within 2 days. ${ }^{24}$ Whereas the results are not as dramatic as those seen in conventional ablative laser resurfacing, significant improvement in rhytides, pigmentation, and laxity of the skin has been shown after only one to two treatments with the fractional $\mathrm{CO}_{2}$ laser. $^{24}$

\section{CONCLUSION}

For each patient, the term "facial rejuvenation" has a unique meaning. The physician should try to fully understand the patient's goals of treatment and their tolerance for downtime, pain, cost, and risk before embarking on any procedure. Although many procedures for facial rejuvenation exist, there is significant variability in cost, recovery time, and efficacy, such that treatment selection must be individualized for each patient. Of course, a critical factor in determining the appropriateness of a facial rejuvenation procedure is physician training and comfort with the procedure. Only procedures that the clinician has been adequately prepared to perform should be offered to the patient.

The first techniques developed for facial skin rejuvenation were in the form of fully ablative procedures. Fully ablative procedures often give the most dramatic improvement in photodamage but also carry the highest risks and longest patient recovery time. The results of these ablative techniques are proportionate to the depth of penetration into the skin, as are patient side effects and downtime. Of the fully ablative techniques for skin rejuvenation, ablative laser resurfacing remains the gold standard due to its unique ability to precisely control the laser depth of penetration and hence the depth of ablation. Although ablative laser resurfacing can give dramatic facial rejuvenation results, this modality still carries significant patient downtime and risks of serious side effects. Additionally, laser ablative resurfacing typically requires general anesthesia and may be quite painful for patients.

Researchers have developed numerous nonablative alternatives for rejuvenation that aim to promote collagen regrowth. These therapies target dermal collagen while protecting the epidermis. The most commonly used of the procedures is the IPL system because it simultaneously targets both erythema and dyspigmentation, two of the typical concerns of patients with photoaging. Whereas these procedures do carry significantly less patient morbidity, their results are not comparable with those of ablative laser resurfacing.

Attempts to bridge the gap between the very efficacious fully ablative therapies with significant associated patient morbidity and the less efficacious nonablative therapies with minimal associated patient morbidity led to the development of nonablative fractional photothermolysis. This procedure has shown promising results with very few patient adverse events. However, numerous treatment sessions are frequently required to achieve the desired results, and the results have not matched those seen in fully ablative laser therapies. Therefore, fully ablative fractional photothermolysis has been developed. This technique most closely re-creates the results seen in fully ablative laser therapy but only requires 1 to 2 days of open wound care. The cosmetic outcome, patient morbidity, and risk of side effects are all proportionate to the percentage of skin treated with MTZs; as a higher percentage of the skin is ablated, the procedure begins to mimic fully ablative laser resurfacing.

There continues to be a significant desire for facial rejuvenation, and physicians continually strive to optimally meet that demand. A promising current trend is to match the effects of ablative resurfacing with a minimal number of treatment sessions and patient morbidity. Although the ablative fractional lasers are the closest technology currently available to achieve that goal, this treatment modality requires continued study, and, undoubtedly, newer technologies will continue to emerge.

\section{REFERENCES}

1. Rabe JH, Mamelak AJ, McElgunn PJ, Morison WL, Sauder DN. Photoaging: mechanisms and repair. J Am Acad Dermatol 2006;55:1-19 
2. Yaar M, Gilchrest BA. Photoageing: mechanism, prevention and therapy. Br J Dermatol 2007;157:874-887

3. Sadick NS, Karcher C, Palmisano L. Cosmetic dermatology of the aging face. Clin Dermatol 2009;27:s3-s12

4. González S, Fernández-Lorente M, Gilaberte-Calzada Y. The latest on skin photoprotection. Clin Dermatol 2008;26: 614-626

5. Serri R, Iorizzo M. Cosmeceuticals: focus on topical retinoids in photoaging. Clin Dermatol 2008;26:633-635

6. Draelos ZD. The cosmeceutical realm. Clin Dermatol 2008; 26:627-632

7. Rubin MG. Manual of Chemical Peels. Philadelphia, PA: JB Lippincott; 1992:5

8. Clark E, Scerri L. Superficial and medium-depth chemical peels. Clin Dermatol 2008;26:209-218

9. Landau M. Chemical peels. Clin Dermatol 2008;26:200-208

10. Ditre CM, Griffin TD, Murphy GF, et al. Effects of alphahydroxy acids on photoaged skin: a pilot clinical, histologic, and ultrastructural study. J Am Acad Dermatol 1996;34 (2 Pt 1):187-195

11. Kligman D, Kligman AM. Salicylic acid peels for the treatment of photoaging. Dermatol Surg 1998;24:325-328

12. Khunger N, Sarkar R, Jain RK. Tretinoin peels versus glycolic acid peels in the treatment of melasma in dark-skinned patients. Dermatol Surg 2004;30:756-760; discussion 760

13. Monheit GD. The Jessner's-trichloroacetic acid peel. An enhanced medium-depth chemical peel. Dermatol Clin 1995; 13:277-283

14. Brody HJ, Hailey CW. Medium-depth chemical peeling of the skin: a variation of superficial chemosurgery. J Dermatol Surg Oncol 1986;12:1268-1275
15. Coleman WP III, Futrell JM. The glycolic acid trichloroacetic acid peel. J Dermatol Surg Oncol 1994;20:76-80

16. Spencer JM, Kurtz ES. Approaches to document the efficacy and safety of microdermabrasion procedure. Dermatol Surg 2006;32:1353-1357

17. Spencer JM. Microdermabrasion. Am J Clin Dermatol 2005; 6:89-92

18. Emsen IM. A different and cheap method: sandpaper (manual dermasanding) in treatment of periorbital wrinkles. J Craniofac Surg 2008;19:812-816

19. Janik JP, Markus JL, Al-Dujaili Z, Markus RF. Laser resurfacing. Semin Plast Surg 2007;21:139-146

20. Fodor L, Carmi N, Fodor A, Ramon Y, Ullmann Y. Intense pulsed light for skin rejuvenation, hair removal, and vascular lesions: a patient satisfaction study and review of the literature. Ann Plast Surg 2009;62:345-349

21. Alexiades-Armenakas MR, Dover JS, Arndt KA. Laser therapy. In: Bolognia JL, Jorizzo JL, Rapini RP, eds. Dermatology. London: Mosby; 2008:2099-2120

22. Goldberg DJ. Photodynamic therapy in skin rejuvenation. Clin Dermatol 2008;26:608-613

23. Sukal SA, Geronemus RG. Thermage: the nonablative radiofrequency for rejuvenation. Clin Dermatol 2008;26: 602-607

24. Jih MH, Kimyai-Asadi A. Fractional photothermolysis: a review and update. Semin Cutan Med Surg 2008;27: 63-71

25. Wanner M, Tanzi EL, Alster TS. Fractional photothermolysis: treatment of facial and nonfacial cutaneous photodamage with a 1,550-nm erbium-doped fiber laser. Dermatol Surg 2007;33:23-28 